\title{
Putting to a bigger hole: Golf performance relates to perceived size
}

\author{
JESSICA K. WITT \\ Purdue University, West Lafayette, Indiana \\ AND \\ Sally A. Linkenauger, Jonathan Z. Bakdash, and Dennis R. Proffitt \\ University of Virginia, Charlottesville, Virginia
}

\begin{abstract}
When people are engaged in a skilled behavior, such as occurs in sports, their perceptions relate optical information to their performance. In the present research, we demonstrate the effects of performance on size perception in golfers. We found that golfers who played better judged the hole to be bigger than did golfers who did not play as well. In follow-up laboratory experiments, participants putted on a golf mat from a location near or far from the hole and then judged the size of the hole. Participants who putted from the near location perceived the hole to be bigger than did participants who putted from the far location. Our results demonstrate that perception is influenced by the perceiver's current ability to act effectively in the environment.
\end{abstract}

Performance affects the perceived size of an action's target. For example, softball players who are hitting well judge the ball to be bigger than do players who have more difficulty hitting (Witt \& Proffitt, 2005). The notion that action-related information such as performance levels affects perception challenges many past and current theories of perception (e.g., Fodor, 1983; Pylyshyn, 2003). Most theories consider perception to be an encapsulated process that is informed solely by optical information and oculomotor adjustments. However, a growing body of research demonstrates that action abilities affect perceived size (Wesp, Cichello, Gracia, \& Davis, 2004; Witt \& Proffitt, 2005), distance (Proffitt, Stefanucci, Banton, \& Epstein, 2003; Witt \& Proffitt, in press; Witt, Proffitt, \& Epstein, 2004, 2005), and geographical slant (Bhalla \& Proffitt, 1999).

In a study on softball players (Witt \& Proffitt, 2005), we found a significant correlation between batting average for the game or games played on that night and judged ball size. Players who had hit well recalled the size of the softball to be bigger than did those who hit less well, thereby suggesting a relationship between perception and performance. However, a question remains as to who really sees the ball as bigger. It could be the people who are more accomplished players, or it could be the people who played better on that night. This question addresses the nature of the effects of performance on perception. These effects might be time dependent, in which case only players who are playing well at the moment will see the ball as bigger, or the effect might be quite general, in which case better players always see the ball as bigger, independent of how they are playing at any given time.
To better understand the effects of performance on perception, we conducted a similar experiment with golfers. Golfers often comment on how their perception of the hole varies with performance. Anecdotal and highly exaggerated comments found in the sport's press suggest that on good days, the hole can look as big as a bucket or a basketball hoop. On bad days, the hole can look as small as a dime, an aspirin, or the inside of a donut. The optical information received by the eye is obviously the same regardless of how well golfers are playing, so do golfers really see the hole differently depending on their performance? And if so, is the effect due to their performance on that day or to their general abilities to play golf? Either way, the results would suggest that the perceived capacity to successfully perform a goal-oriented action can influence how big the target looks.

\section{EXPERIMENT 1}

We recruited golfers after they played a round of golf and asked them to estimate the size of the hole. We also collected information on how well they played that day, and found correlations between performance and apparent hole size.

\section{Method}

Participants. Forty-six golfers ( 1 female; age range 26-66, mean age $=45.9$ ) at the Providence Golf Club in Richmond, VA, agreed to participate. All gave informed consent.

Stimuli. Nine black paper circles were glued to a piece of white poster board that was $76 \mathrm{~cm}$ wide and $51 \mathrm{~cm}$ tall with the smaller circles in the top left corner and the larger ones on the bottom right.

J. K.Witt, jkwitt@purdue.edu 
Diameters ranged unsystematically from 9 to $13 \mathrm{~cm}$. The diameter of a golf hole is $10.8 \mathrm{~cm}$.

Procedure. After they had played a full round of golf, players were recruited to participate in the experiment. They signed an informed consent agreement. They were shown the poster board with the various circles and were asked to pick the circle that they thought best corresponded to the size of the hole. Then, we collected information on their score for the course that day, their handicap, how many putts they took on the 18th green, and how many strokes they took on the 18th hole. For each of these measures, a lower score indicates better performance or ability. Handicap is a number computed in golf that is an assessment of a golfer's ability. It is calculated as the mean difference between a player's score minus the par score for the course. For example, a player who typically gets a 74 on courses with pars of 72 has a handicap of 2 . Lower handicaps signify better players.

We also obtained three subjective reports on the participants' performance. Participants rated their putting abilities in comparison with others who shared their handicap, putting on that day relative to their regular putting abilities, and play on that day relative to their regular playing abilities on a scale from 1 to 7 , where a 1 meant that they had their best day and a 7 meant that they had their worst.

\section{Results and Discussion}

As is shown in Figure 1, players having lower scores on the course that day judged the hole to be bigger than did players who had higher scores $(r=-.30, N=46$, $p=.02$, one-tailed, Spearman rank-order correlation). In contrast, handicap, a measure of longer term playing ability, was not significantly correlated with judged hole size $(r=-.19, N=38, p=.12$, one-tailed, Spearman rankorder correlation). ${ }^{1}$ Recall that a lower handicap signifies a better player. Combined, these results suggest that better players did not perceive the hole as being bigger, but players that were playing better on that given day did perceive the hole as bigger.
Furthermore, players who took fewer putts to get the ball in the 18th hole judged it to be bigger than did players who took more putts $(r=-.26, p=.04$, one-tailed, Spearman rank-order correlation). The correlation between total strokes on the 18th hole and its judged size was not significant ( $p=.14$ ), implying that this effect was specific to putting performance, for which hole size is relevant. There were no significant correlations with any of our subjective measures of performance ( $p \mathrm{~s}>.2$ ), so self-assessed performance did not relate to apparent hole size, whereas actual performance did.

Our results expand on earlier research with softball players where we showed that batters who were hitting better on a given day recalled the softball to be bigger (Witt \& Proffitt, 2005). In this experiment, golfers who were playing better judged the size of the hole to be bigger, thus demonstrating another link between perception and performance. Although we found a significant correlation between perception of hole size and golf performance on that day (as assessed by course score), we did not find a significant correlation between perception of hole size and how good a player is (as assessed by handicap). This result implies that a highly skilled player such as Tiger Woods does not always see the hole as bigger just because he is a terrific player, but rather, any person may perceive the hole as being bigger when he or she is playing well. However, a nonsignificant correlation is difficult to interpret, and a significant correlation might have been found with more participants or a wider range of handicaps. In our experiment, handicap ranged from 7 to 36 (range $=$ 29), whereas course score ranged from 77 to 123 (range $=$ 46). Because we measured only day-of performance in the

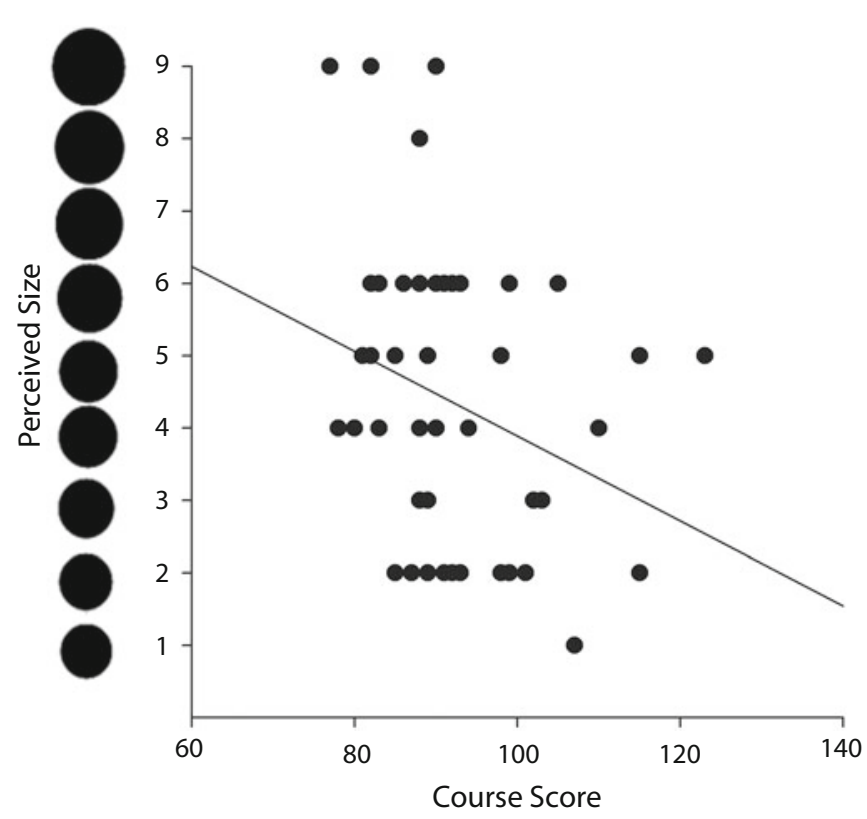

Figure 1. Apparent size of a golf hole as a function of score on the course that day. Each circle represents 1 or more participants' data. The circles on the $y$-axis are drawn to preserve relative size of the stimuli. The solid line is the correlation between course score and the circle selected as best matching the size of the hole. 
softball study as well (Witt \& Proffitt, 2005), the present experiment raises an interesting question: What aspects of performance and performance capacity are related to perception? In future studies, we plan to include longitudinal measures to see whether perceived size changes for a player of a given handicap as daily performance levels rise and fall.

We also found that apparent hole size was correlated with putting performance on the last hole but not with overall performance on the last hole, suggesting that these effects are specific to the relevant task. Finally, apparent size is not related to subjective measures of performance. Players who think they are playing better do not necessarily recall the hole as appearing bigger.

\section{EXPERIMENT 2}

As with the softball study (Witt \& Proffitt, 2005), we cannot be sure whether performance influenced perceived hole size or remembered hole size, because participants estimated size from memory. The next two studies addressed this question. In Experiment 2, we sought to replicate the effect of performance on remembered hole size in a laboratory context. Experiment 3 differed from Experiment 2 only in that size judgments were made while the hole was in view.

We placed a store-purchased practice putting mat in the laboratory. Putting performance was manipulated by having some participants putt from very close to the hole and having others putt from farther away. We tested whether participants in the easy (close) condition would judge the hole to be bigger than would participants in the hard (far) condition.

\section{Method}

Participants. Forty (15 male, 25 female) University of Virginia students, ranging in age from 18 to 34 , participated in this experiment either for a snack or to fulfill a research requirement for course credit. All participants indicated that they would play golf righthanded and had normal or corrected-to-normal vision. All gave informed consent.

Stimuli and Materials. Participants chose one of two righthanded golf putters, which differed in length $(0.89$ and $0.93 \mathrm{~m}$ long). The putting mat was $2.15 \mathrm{~m}$ long and $0.34 \mathrm{~m}$ wide. The mat sloped upward at a $10^{\circ}$ angle starting $0.42 \mathrm{~m}$ before the hole. The hole was $9.52 \mathrm{~cm}$ in diameter. Estimated hole size was collected in Microsoft Paint using a standard computer mouse and keyboard.

Procedure. Participants were assigned to either the easy or the hard condition in alternating order. For the hard condition, participants stood $2.15 \mathrm{~m}$ away from the front of the putting hole, and for the easy condition, participants stood $0.4 \mathrm{~m}$ away from the hole. Participants were instructed that, after some practice, they would putt the ball 10 times. We asked participants to predict how many putts they would make. We told them that if they made as many as they predicted, they would receive their choice of a snack. They were given $2 \mathrm{~min}$ alone to practice putting before attempting their putts. After the participants had practiced, the experimenter told the participants to take 10 putts. Upon completion of their putts, all participants were given their choice of snacks, regardless of whether they made their predicted number of putts.

Participants were then directed into another room to perform a visual matching task. They were instructed to sit in front of a Dell laptop running Microsoft Paint, a simple drawing program that consists of a sketching area and several types of drawing tools such as a paintbrush or shape tools. The sketching area was always blank to start. Participants used the ellipse tool by holding down the shift key and dragging the mouse to draw a filled-in black circle. They were instructed to draw the circle to match the actual physical size of the putting hole. They were encouraged to redraw until they were satisfied that their circle matched the size of the hole.

\section{Results and Discussion}

Not surprisingly, participants in the easy condition $(M=60.0 \%, S E=5.13 \%)$ predicted making more putts than did those in the hard condition $(M=38.3 \%, S E=$ $3.44 \%)[t(38)=3.52, p<.01$, one-tailed, $d=0.55]$. Also, a higher percentage of putts was made in the easy condition $(M=73.5 \%, S E=4.06 \%)$ than in the hard condition $(M=24.5 \%, S E=4.38 \%)[t(38)=8.206, p<$ .01 , one-tailed, $d=1.30]$.

More interestingly, participants in the easy condition drew the circle bigger than did participants in the hard condition (see Figure 2) $[t(38)=1.69, p=.05$, onetailed, $d=0.27]$. This result suggests that participants in the easy condition perceived the hole to be bigger than did participants in the hard condition, so they drew larger circles as matching the size of the hole. Because putting is more difficult from a farther distance and performance was markedly worse in the hard condition relative to the easy condition, these findings suggest that putting performance influences apparent hole size.

However, there are two concerns with this experiment. First, participants had different views of the hole while putting. Participants in the easy condition were closer to the hole, so the hole subtended a larger visual angle and exhibited less projected compression in its aspect ratio. These different viewpoints may account for differences in apparent hole size.

The second concern is that participants did not have vision of the hole when they estimated its size. Thus, the differences in apparent hole size may be due to an effect of

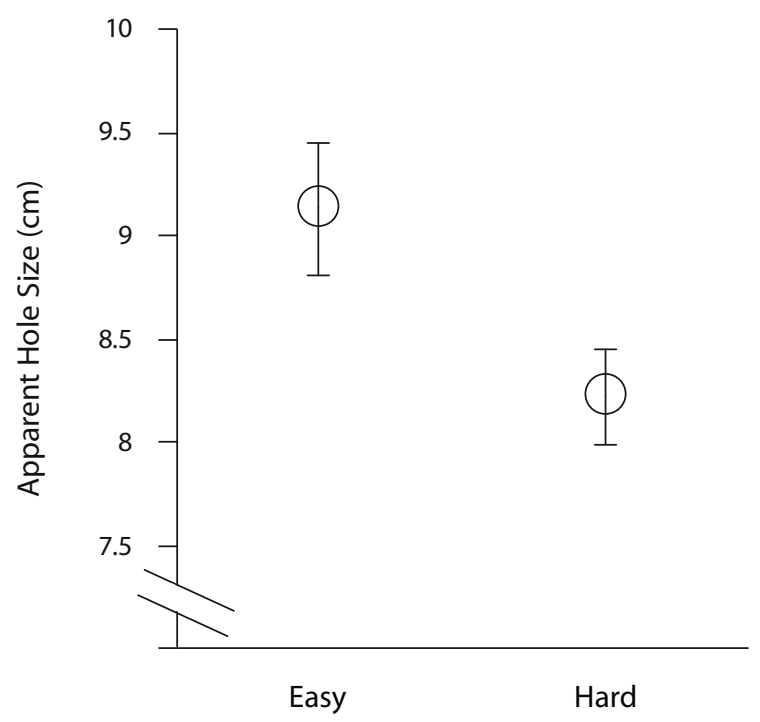

Figure 2. Apparent size of the golf hole in the putting mat by condition in Experiment 2 as measured by drawing circles in Microsoft Paint. The diameter of the hole was $9.52 \mathrm{~cm}$. Participants did not have vision of the hole when making their estimates. Error bars represent one standard error of the mean. 
performance on memory rather than on perception. This critique applies to Experiment 1 and to the finding that batting performance influences apparent ball size (Witt $\&$ Proffitt, 2005). Thus, in the last experiment, we ran the same procedure as in Experiment 2 except that participants viewed the hole while making their size judgments.

\section{EXPERIMENT 3}

As in Experiment 2, participants putted from a near or far distance. However, when these participants made size judgments, they had full vision of the hole. Moreover, all participants judged hole size from the same location and thus had the same viewpoint of the hole while making judgments of its size.

\section{Method \\ Participants. Fifty (21 male, 29 female) University of Virginia students, ranging in age from 17 to 34 , participated in this ex- periment either for a snack or to fulfill a research requirement for course credit. All participants golfed right-handed and had normal or corrected-to-normal vision. All gave informed consent. \\ Stimuli and Materials. The same golf putters and putting mat were used as in Experiment 2. Estimated hole size was collected in Microsoft Paint using a standard computer mouse and keyboard. \\ Procedure. The procedure was the same as in Experiment 2 except that participants viewed the hole while estimating its size. After par- ticipants finished putting, they were instructed to sit in front of a Dell desktop computer, which was situated beside the golf mat, approxi- mately $1 \mathrm{~m}$ to the right of the putting hole. Participants were instructed to use the ellipse tool in Microsoft Paint to draw a circle that was the same size as the putting hole. Participants were allowed to look back and forth between their drawing and the hole as much as they liked, and they were encouraged to redraw the circle until they were satisfied that the circle on the screen matched the size of the hole.}

\section{Results and Discussion}

As in Experiment 2, participants predicted making more putts in the easy condition $(M=60.00 \%, S E=$ $3.92 \%)$ than in the hard condition $(M=36.80 \%, S E=$ $3.25 \%)[t(48)=4.56, p<.01$, one-tailed, $d=0.65]$. A higher percentage of putts was made in the easy condition $(M=68.8 \%, S E=4.45 \%)$ than in the hard condition $(M=28.00 \%, S E=3.32 \%)[t(48)=7.36, p<.01$, onetailed, $d=1.04]$.

Participants in the easy condition drew the circle bigger than did the participants in the hard condition $[t(48)=$ $1.73, p=.05$, one-tailed, $d=0.24$ ] (see Figure 3). Even when participants had full vision of the hole, they perceived its size differently. Thus, putting performance influences perceived hole size and not just remembered hole size.

Participants in the two groups viewed the hole from different distances while putting, so a potential concern is that the difference in viewing distance is responsible for our effects. The present studies do not directly speak to this issue. However, in Experiment 3, both groups viewed the hole from the same distance when they judged size, so the viewing perspective at the time when we collected our dependent measure was the same for both groups. Thus, our effects are likely due to differences in the putting task and not to differences in viewing distance. An effect due solely to viewing distance would produce results contrary

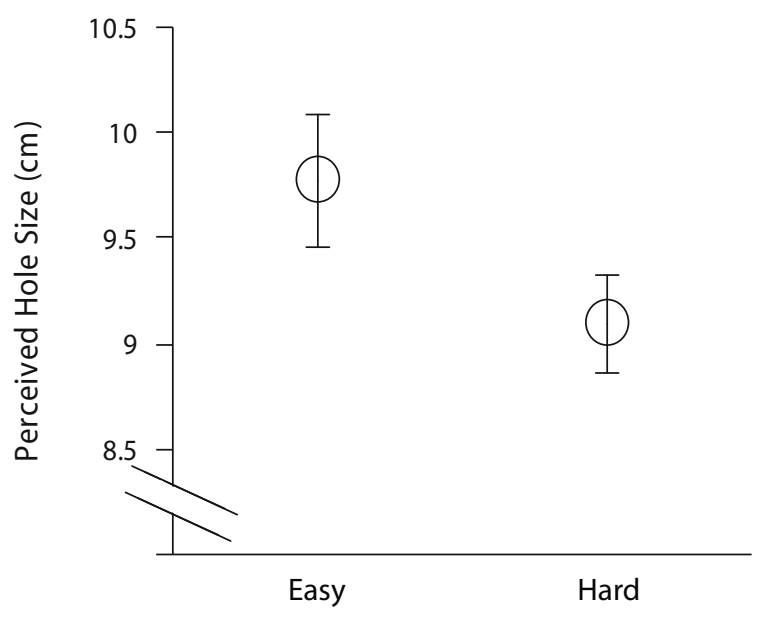

Figure 3. Perceived size of the golf hole in the putting mat by condition in Experiment 3 as measured by drawing circles in Microsoft Paint. The diameter of the hole was $9.52 \mathrm{~cm}$. Participants had vision of the hole when making their estimates. Error bars represent one standard error of the mean.

to the findings of Experiment 1. Better golfers can typically make putts from farther away and the better golfers probably viewed the hole from a farther distance, yet they perceived the hole to be bigger.

\section{GENERAL DISCUSSION}

In three experiments, we demonstrated that golfers perceive the size of the hole in relation to their golfing performance. In the first experiment, we asked golfers after they played a full round of golf to judge the size of the golf hole. We found that judged hole size was negatively correlated with the players' performance that day. Players who played better judged the hole to be bigger. This finding is consistent with our early finding that softball players who are batting better judge the ball as being bigger (Witt \& Proffitt, 2005).

However, although course score correlated with apparent hole size, handicap did not. Thus, perception may not be a function of how good a player is, but rather of how well that player is playing at that specific moment. Our sample of golfers was limited to relatively unskilled golfers, so we are not sure that this statement would generalize over the wider range of abilities of very skilled or professional golfers. In Experiment 1, the mean handicap was 18.2, and in Experiments 2 and 3, only 38\% of all participants had experience with golf. It would be interesting to see how golfers' perceptions of the hole change from day to day and whether the variation in perception differs across levels of expertise.

Apparent cup size correlated with putting performance on the last hole but not with overall performance on the last hole, suggesting that these effects are specific to the relevant task. The data from the last hole suggest that the apparent size of the hole is influenced only by performance on tasks that directly involve the hole. In this case, the only relevant task involving the hole's size consisted 
of putting, not driving or hitting. Except for seemingly miraculous shots in which the ball happens to go into the cup from outside of the putting green, hole size becomes relevant only when putting. Consequently, performance on strokes other than putting ought not to contribute to the apparent size of the golf cup.

Finally, in these studies, apparent size was not related to subjective measures of performance. Players who thought they were playing better did not necessarily report the hole as appearing bigger. Only actual performance affected the recalled size of the golf cup. As a result, the apparent size of the cup may be independent of several traits of the player, including, perhaps, their confidence, optimism, or general attitude toward themselves. Because these traits can affect performance (Stone, Lynch, Sjomeling, \& Darley, 1999), one might think they would have also affected perception. However, more detailed experiments would be needed before making any definite claims about the relationship between attitude and perception.

In the last two experiments, university students putted golf balls on a putting mat from a location close to the hole or one that was far from it. Then they judged the size of the hole either from memory (Experiment 2) or while still viewing the hole (Experiment 3 ). In both experiments, participants who putted from the closer location drew the matching circle to be bigger than participants who putted from the far location. The results of Experiment 3 demonstrate that people's putting performance can affect their perception of the hole's size, as opposed to just inducing a memory bias.

Although these results suggest that a relationship exists between performance and perception, the causal direction of this finding is unclear. For example, do golfers putt better and, therefore, see the hole as bigger, or do they see the hole as bigger and therefore putt better? The present experiments do not speak to this question; however, we speculate that the relationship is reciprocal in such a way that perception and performance likely influence each other.

Our findings are consistent with other research showing effects of action potential on perception. Targets placed just beyond arm's reach look closer when a perceiver intends to reach with a tool than when the perceiver intends to reach without the tool (Witt \& Proffitt, in press; Witt et al., 2005). Hills look steeper (Bhalla \& Proffitt, 1999) and distances look farther (Proffitt et al., 2003) when the perceiver wears a heavy backpack and thus will have to exert more energy to walk. Similarly, targets look farther away to people when they have to throw a heavy ball compared with a light ball (Witt et al., 2004). In all of these experiments, the optical information was held constant, yet perception varied depending on the perceiver's ability to perform the intended action.

In summary, our results demonstrate that people's perceptions of target size are scaled by their current abilities to act effectively on the target. In turn, golfers who are playing better see the hole as bigger than do golfers who are not playing as well.

\section{AUTHOR NOTE}

This research was supported by NIH Grant RO1MH075781-01A2 to D.R.P. The authors thank Ray Ryan and the Providence Golf Club for their assistance with data collection. Correspondence concerning this article should be addressed to J. K. Witt, Department of Psychological Sciences, Purdue University, 703 Third Street, West Lafayette, IN 47907 (e-mail: jkwitt@purdue.edu).

\section{REFERENCES}

Bhalla, M., \& Proffitt, D. R. (1999). Visual-motor recalibration in geographical slant perception. Journal of Experimental Psychology: Human Perception \& Performance, 25, 1076-1096.

FoDOR, J. A. (1983). Modularity of mind: An essay on faculty psychology. Cambridge, MA: MIT Press.

Proffitt, D. R., Stefanucci, J., Banton, T., \& Epstein, W. (2003). The role of effort in distance perception. Psychological Science, 14, 106-112.

Pylyshyn, Z. W. (2003). Seeing and visualizing: It's not what you think. Cambridge, MA: MIT Press.

Stone, J., Lynch, C. I., Sjomeling, M., \& Darley, J. M. (1999). Stereotype threat effects on black and white athletic performance. Journal of Personality \& Social Psychology, 77, 1213-1227.

Wesp, R., Cichello, P., Gracia, E. B., \& Davis, K. (2004). Observing and engaging in purposeful actions with objects influences estimates of their size. Perception \& Psychophysics, 66, 1261-1267.

Witt, J. K., \& ProffitT, D. R. (2005). See the ball, hit the ball: Apparent ball size is correlated with batting average. Psychological Science, 16, 937-938.

Witt, J. K., \& Proffitt, D. R. (in press). Action-specific influences on distance perception: A role for motor simulation. Journal of Experimental Psychology: Human Perception \& Performance.

Witt, J. K., Proffitt, D. R., \& Epstein, W. (2004). Perceiving distance: A role of effort and intent. Perception, 33, 577-590.

Witt, J. K., Proffitt, D. R., \& Epstein, W. (2005). Tool use affects perceived distance, but only when you intend to use it. Journal of Experimental Psychology: Human Perception \& Performance, 31, 880-888.

\section{NOTE}

1. Eight participants did not have a handicap or did not report theirs.

(Manuscript received May 23, 2007; revision accepted for publication December 4, 2007.) 